\title{
Serum metabolomic and lipidomic profiling identifies diagnostic biomarkers for seropositive and seronegative rheumatoid arthritis patients
}

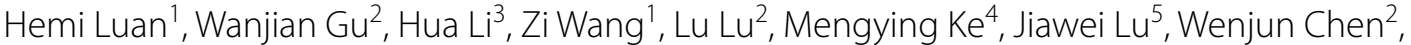

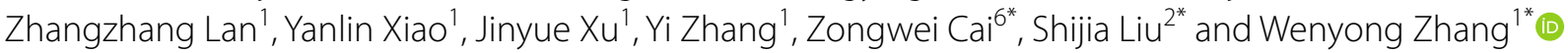

\begin{abstract}
Background: Diagnosing seronegative rheumatoid arthritis (RA) can be challenging due to complex diagnostic criteria. We sought to discover diagnostic biomarkers for seronegative RA cases by studying metabolomic and lipidomic changes in RA patient serum.

Methods: We performed comprehensive metabolomic and lipidomic profiling in serum of 225 RA patients and 100 normal controls. These samples were divided into a discovery set $(n=243)$ and a validation set $(n=82)$. A machinelearning-based multivariate classification model was constructed using distinctive metabolites and lipids signals.

Results: Twenty-six metabolites and lipids were identified from the discovery cohort to construct a RA diagnosis model. The model was subsequently tested on a validation set and achieved accuracy of $90.2 \%$, with sensitivity of $89.7 \%$ and specificity of $90.6 \%$. Both seropositive and seronegative patients were identified using this model. A cooccurrence network using serum omics profiles was built and parsed into six modules, showing significant association between the inflammation and immune activity markers and aberrant metabolism of energy metabolism, lipids metabolism and amino acid metabolism. Acyl carnitines (20:3), aspartyl-phenylalanine, pipecolic acid, phosphatidylethanolamine PE (18:1) and lysophosphatidylethanolamine LPE (20:3) were positively correlated with the RA disease activity, while histidine and phosphatidic acid PA (28:0) were negatively correlated with the RA disease activity.
\end{abstract}

Conclusions: A panel of 26 serum markers were selected from omics profiles to build a machine-learning-based prediction model that could aid in diagnosing seronegative RA patients. Potential markers were also identified in stratifying RA cases based on disease activity.

Keywords: Rheumatoid arthritis, Seronegative, Metabolomic, Lipidomic

*Correspondence: zwcai@hkbu.edu.hk; liushijia2011@163.com; zhangwy@sustech.edu.cn

1 School of Medicine, Academy for Advanced Interdisciplinary Studies,

Southern University of Science and Technology, 1088 Xueyuan Rd.

Shenzhen, China

${ }^{2}$ Affiliated Hospital of Nanjing University of Chinese Medicine,

Nanjing 210029, Jiangsu, China

${ }^{6}$ State Key Laboratory of Environmental and Biological Analysis (SKLEBA)

Department of Chemistry, Hong Kong Baptist University, Kowloon Tong, Hong Kong, China

Full list of author information is available at the end of the article

\section{Introduction}

Rheumatoid arthritis (RA) is a complex chronic autoimmune disease with variable presenting symptoms and serum autoantibody test results. Currently diagnosing RA is primarily based on clinical symptoms and the presence of various serum autoantibodies including rheumatoid factor (RF) and anti-citrullinated protein antibody (ACPA) [1-3]. Although most patients with confirmed RA have an abnormal test for RF and/ or ACPA, about $15 \%-20 \%$ of cases do not have the elevated levels of RF original author(s) and the source, provide a link to the Creative Commons licence, and indicate if changes were made. The images or other third party material in this article are included in the article's Creative Commons licence, unless indicated otherwise in a credit line to the material. If material is not included in the article's Creative Commons licence and your intended use is not permitted by statutory regulation or exceeds the permitted use, you will need to obtain permission directly from the copyright holder. To view a copy of this licence, visit http://creativecommons.org/licenses/by/4.0/. The Creative Commons Public Domain Dedication waiver (http://creativeco mmons.org/publicdomain/zero/1.0/) applies to the data made available in this article, unless otherwise stated in a credit line to the data. 
and ACPA [4]. Early recognition and assessment of the progression of RA are of paramount importance to avoid the irreversible damage. Therefore, developing reliable diagnostic biomarkers for evaluating seronegative RA is needed [1].

Chronic inflammatory state in RA causes metabolic changes that can serve as biomarkers for diagnosis, disease activity and treatment efficacy monitoring $[5,6]$. The liquid chromatography-mass spectrometry (LC-MS)based metabolomic and lipidomic approach is a powerful tool to discover potential biomarkers and metabolic pathway remodeling occurred in chronic diseases including RA $[4,7,8]$. However, small sample size and inadequate number of targets studied so far limit definitive conclusions drawing [4, 9-13]. The potential of using LC-MS-based metabolomic and lipidomic profiling for novel biomarker discovery to achieve more accurate diagnosis and disease activity monitoring remains largely unexplored.

In our study, we aimed to build a classification model that can be applied to both seropositive and seronegative patients by integrative LC-MS-based metabolomic and lipidomic profiling of serum samples from a large cohort of RA patients and normal control subjects.

\section{Materials and methods Clinical samples collection}

Serum samples were collected from 225 RA patients and 100 normal control subjects at the Affiliated Hospital of Nanjing University of Chinese Medicine. The clinical and demographic characteristics of the study were summarized in Table 1. Disease activity for RA patients was assessed at the time of serum collection through quantification of tender and swollen joints, erythrocyte sedimentation rate (ESR), C-reactive protein (CRP) levels and the visual analog scale-grading of the patients. Cytokine concentrations were determined by immunoassay. Subjects in any one or more of the following categories were excluded from our analysis: (1) the presence of type I or II diabetes; (2) active viral and/or bacterial infection; (3) the presence of osteoarthritis. The study was approved by the medical ethics committee of the Affiliated Hospital of Nanjing University of Chinese Medicine and followed the tenets of the Declaration of Helsinki (2018NL-10602). Written informed consents were obtained from all study subjects. Patients were clinically diagnosed with RA according to the American College of Rheumatology and European League Against Rheumatism (EULAR) 2010 criteria $[9,14]$. Venous blood was collected in the morning before breakfast from all the participants, and then sera were separated and stored at $-80{ }^{\circ} \mathrm{C}$ until use.
Table 1 Clinical and demographic characteristics of the study participants

\begin{tabular}{llll}
\hline & $\begin{array}{l}\text { Normal } \\
\text { controls } \\
(\mathbf{n}=\mathbf{1 0 0})\end{array}$ & RA (n=225) & p value \\
& $69: 31$ & $189: 36$ & \\
\hline Female: Male & $44.32(21-66)$ & $57.71(27-87)$ & $<0.001$ \\
Age (years) & $2.6(0.5-6.0)$ & $19.3(1.0-127.0)$ & 0.003 \\
CRP (IU/mL) & $9.7(2.0-24.0)$ & $43.8(2.0-140.0)$ & 0.002 \\
ESR (mm/h) & $2.1(0.9-3.9)$ & $2.6(0.2-6.4)$ & 0.16 \\
IgA (IU/mL) & $12.8(9.4-19.8)$ & $14.2(5.9-50.7)$ & 0.39 \\
IgG (IU/mL) & $1.1(0.4-3.2)$ & $1.3(0.3-4.4)$ & 0.61 \\
IgM (IU/mL) & $9.7(0.4-40)$ & \\
Disease duration (minimum- & - & & \\
maximum in months) & & $3.5(1.0-7.1)$ & \\
DAS28-CRP (minimum-maxi- & - & $4.0(1.2-7.8)$ & \\
mum) & & & \\
DAS28-ESR (minimum-maxi- & - & $57.8 \%$ & \\
mum) & & $42.2 \%$ & \\
Rheumatoid factor positive & - & $49.4 \%$ & \\
ACPA positive & - & $49.4 \%$ & \\
ANA positive & - & - &
\end{tabular}

Statistical significance was determined using unpaired two-tailed Student's $t$ test

ESR, Erythrocyte sedimentation rate; CRP, C-Reactive protein; IgA, Immunoglobulin A; IgG, Immunoglobulin G; IgM, Immunoglobulin M; ACPA, anti-citrullinated protein antibody; ANA, anti-nuclear antibodies; ANA, anti-keratin antibodies; DAS28-CRP, Disease activity score 28-joint count C reactive protein; DAS28-ESR, Disease activity score 28 -joint count erythrocyte sedimentation rate

\section{Sample preparation}

The metabolites and lipids in serum were isolated and treated as previously reported [15] with slight modifications. Fifty microliters of thawed serum samples were precipitated by adding $200 \mu \mathrm{l}$ of cold acetonitrile. After centrifugation at $14,000 \mathrm{~g}$ for $10 \mathrm{~min}$ at $4{ }^{\circ} \mathrm{C}$, the supernatant was divided into four fractions: two for polar metabolites analysis by hydrophilic interaction liquid chromatography (HILIC)-LC-MS methods with positive ion mode electrospray ionization (ESI) and negative ion mode ESI, namely method 1 and method 2, respectively; two for lipids analysis by reverse phases (RP)-LCMS methods with positive ion mode ESI and negative ion mode ESI, namely method 3 and method 4, respectively. After centrifugation, samples were dried and stored at $-80{ }^{\circ} \mathrm{C}$ until use. The quality control (QC) samples were prepared by mixing equal volumes of sera from RA patients and controls before sample preparation as they were aliquoted for analysis. These QC samples were utilized to estimate a "mean" profile representing all analytes encountered during the LC-MS analysis [16]. 


\section{Metabolomic and lipidomic analysis}

For the polar metabolite profiling, dried samples were reconstituted in $100 \mu \mathrm{l}$ of $80 \%$ methanol and analyzed by using a Dionex U3000 LC system coupled online to a Q Exactive Orbitrap MS instrument (Thermo Fisher Scientific, MA, USA) set at 35,000 resolution (at $\mathrm{m} / \mathrm{z} 200$ ). For the lipids profiling, dried samples were reconstituted in $100 \mu \mathrm{l}$ of acetonitrile for instrument analysis. (HILIC)LC-MS and (RP)-LC-MS were performed for metabolomic and lipidomic profiling, respectively. The details of the analytical experiments were described in Supplemental Materials. Data pre-processing was carried out as previously reported [17]. The XCMS package [18] was used for the extraction of peak abundances of metabolites and lipids. The background noises and contaminants in the peak tables were filtered by using CPVA with default parameters [19]. Quality assurance was achieved by the statTarget package [20] as follows. Briefly, peaks with more than $50 \%$ missing values were removed. The intensity of remaining peaks in samples was corrected according to the QC-RFSC algorithm. Principal component analysis (PCA)-based data quality evaluation was provided in Additional file 1: Fig. S1. Polar metabolites identification was according to the MS/MS spectra, the retention time of commercially available standard compounds, and the accurate mass of compounds from the Human Metabolome Database (www.hmdb.ca) and MassBank of North America (http://mona.fiehnlab.ucdav is.edu). Lipids were identified based on MS/MS match by using the LipidSearch software v4.1 (Thermo Fisher Scientific, CA, USA). The parameters in LipidSearch were set as follows: precursor tolerance at $5 \mathrm{ppm}$, product tolerance at $5 \mathrm{ppm}$, product ion threshold at 5\%, and intensity threshold at $1.0 \%$. Quantitation and Toprank filter were turned on. Main node filters were set to Main Isomer Peaks, and ID quality was graded from A-B. Pathway analysis based on identified metabolites was carried out using Metaboanalyst 4.0 [21] according to the Kyoto Encyclopedia of Genes and Genomes (KEGG) pathway database (www.genome.jp/kegg/).

\section{Data analyses}

The Mann-Whitney-Wilcoxon test or Welch's t-test, was performed to measure the significance of each peak, with results adjusted for multiple testing using false discovery rates (FDR) correction. The cross-validated partial least squares discriminant analysis (PLS-DA) and variable importance in the projection $\left(\mathrm{VIP}_{\text {plsda }}\right)$ were calculated using the statTarget package. Serum metabolites passing the threshold of $\mathrm{VIP}_{\text {plsda }}>1$, fold change $>1.2$ or $<0.8$, and adjusted p-value $<0.05$ were regarded as potential markers between the RA and NCs groups. The alluvial plots and forest plot were performed using the ggalluvial package and forestplot package, respectively. The cooccurrence network was carried out and visualized in the igraph package (https://igraph.org). Spearman's rank correlation was used for a measure of correlation between two variables. The nodes represent clinical parameters or metabolites and lipids, and two nodes were connected if they were significantly correlated (adjusted p-value $<0.05$ and $r$-value $>0.2$ ). The ordinal regression was performed by using the ordinalgmifs package for fitting an ordinal response model [22]. Coupling the receiver operating characteristic curve (ROC) with its area under the curve (AUC), a widely used method to estimate the diagnostic potential of a classifier in clinical applications, was performed using the pROC package [23]. All packages were implemented using the freely available R language.

\section{Results}

Clinical data and patient characteristics

The demographic information of the study participants was summarized in Table 1. Among 225 RA patients, the positive rate of RF and ACPA was $57.8 \%$ and $42.2 \%$, respectively. These samples were randomly divided into two independent cohorts. The discovery set consisted of 172 RA patients and 71 normal controls (NCs), and the validation set consisted of $53 \mathrm{RA}$ patients and $29 \mathrm{NCs}$ (Fig. 1A). The alluvial plot shown in Fig. 1B displayed the number of individuals across the disease status, gender, disease activity score (DAS28-CRP and DAS28-ESR), as well as the status of autoantibodies such as rheumatoid factor (RF), ACPA, anti-nuclear antibodies (ANA), and anti-keratin antibodies (AKA). The positive rates of RF, ACPA, ANA, AKA in the RA patients were 57.8\%, 42.2\%, $49.4 \%$ and $49.4 \%$ (Table 1 and Fig. 1B).

\section{Metabolomic and lipidomic profile (MLP)}

In four datasets generated from four different LC-MS runs, there were 1697 and 1960 high-confident peaks identified for polar metabolites with method 1 and 2, and 4771 and 3973 high-confident peaks identified for lipids with method 3 and 4. From this 12401 high-confident peaks we identified 265 metabolites and lipids (Additional file 1: Table S1), including 38 organic acids, 10 amines, 37 amino acids, 5 nucleotides, 4 bile acids, 26 acyl-carnitines (AcCa), 118 glycerophospholipids, 9 sphingolipids and 2 glycerolipids. These metabolites represent enriched metabolic pathways involving carnitine synthesis, oxidation of branched-chain fatty acids, biotin metabolism, malate-aspartate shuttle, citric acid cycle, urea cycle, phenylalanine and tyrosine metabolism, phospholipid biosynthesis and histidine metabolism (Additional file 1: Fig. S2). 


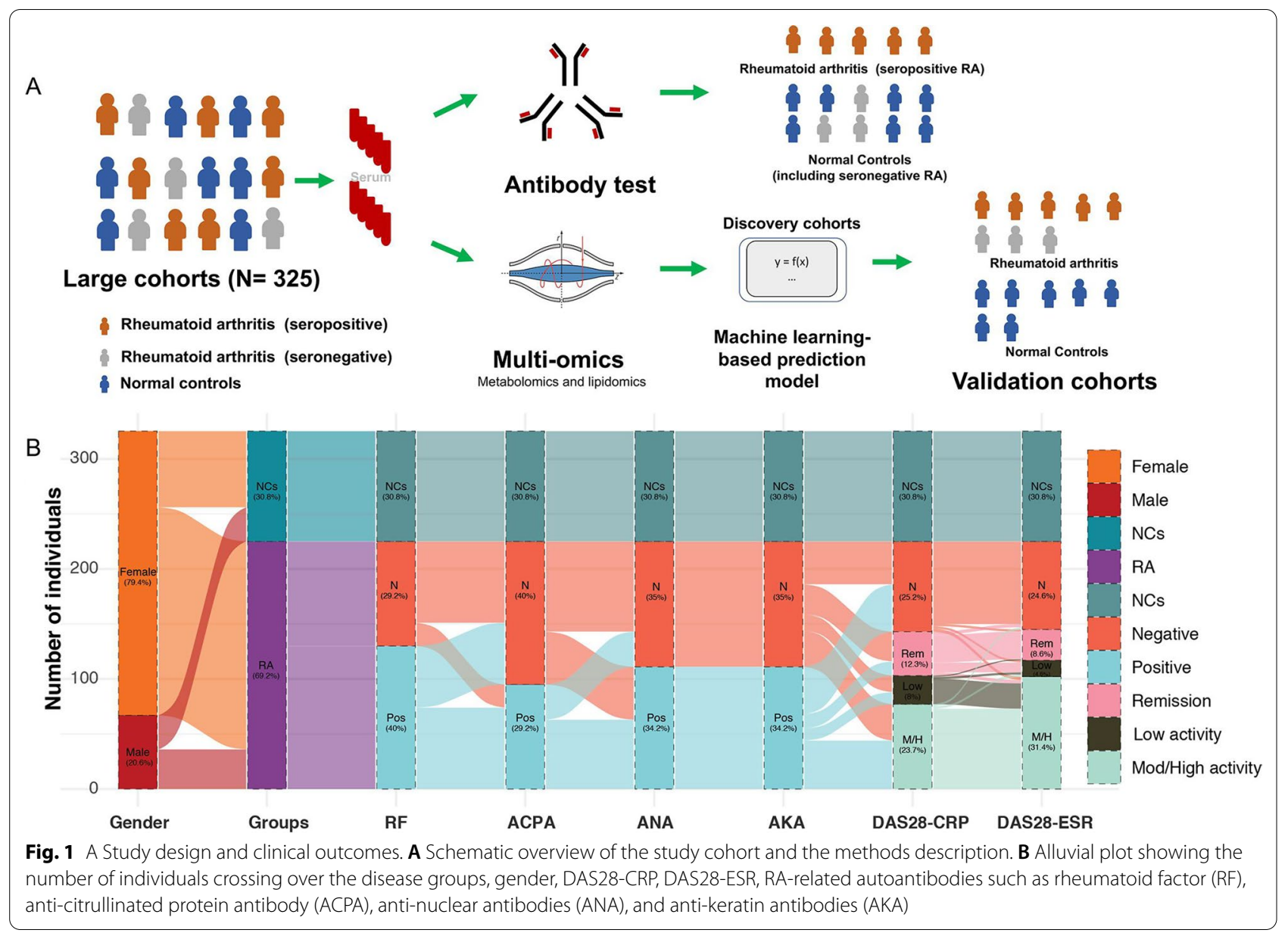

\section{Clustering of test samples using MLP and pathway enrichment analysis}

To analyze whether any of the identified metabolites or lipids are associated with RA, we carried out the univariate and multivariate analysis of the identified metabolites and lipids. The well-established partial least squaresdiscriminant analysis (PLS-DA) demonstrated excellent separation of NCs from seropositive RA and seronegative RA based on MLP (Fig. 2A). The permutation tests $(\mathrm{n}=1000)$ were performed to validate the PLS-DA model we used (Additional file 1: Fig. S3). This separation clearly demonstrated the difference in serum metabolite and lipid levels that existed between RA patients and normal control subjects. Statistical analysis identified 68 significantly upregulated and 38 downregulated serum metabolites and lipids that were correlated with RA (Fig. 2B). Pathway enrichment analysis (Fig. 2C) highlighted the top enriched metabolic pathways in RA. Specifically, metabolic products associated with Warburg effect, pentose phosphate metabolism, glycolysis, and lipid metabolism products involving phospholipid, sphingolipid, oxidation of branched chain fatty acids and carnitine synthesis were upregulated in the RA group, while histidine metabolism was downregulated.

\section{Constructing a multivariate classification model for RA}

We constructed a multivariate classification model including 26 metabolites and lipids that could separate seropositive and seronegative RA cases from normal controls (Fig. 2D, E). We evaluated three machine learning algorithms such as binary logistic regression, random forest and support vector machine on the above-mentioned metabolites and lipids for the classification of RA cases using the discovery set $(n=243)$. The binary logistic regression algorithm based model trained with leave-one-out cross-validation had an accuracy of $100 \%$ (Fig. 2E, AUC =1.00). This model was tested using the independent validation set $(n=82$, Additional file 1 : Table S2), and showed AUC $=0.91$ with test sensitivity of $89.7 \%$ and specificity of $90.6 \%$ (Fig. 2E). We did an analysis of the 5 misclassified RA cases, and it is noteworthy that all of the 5 cases had borderline to positive RF antibody levels (Additional file 1: Table S3). 


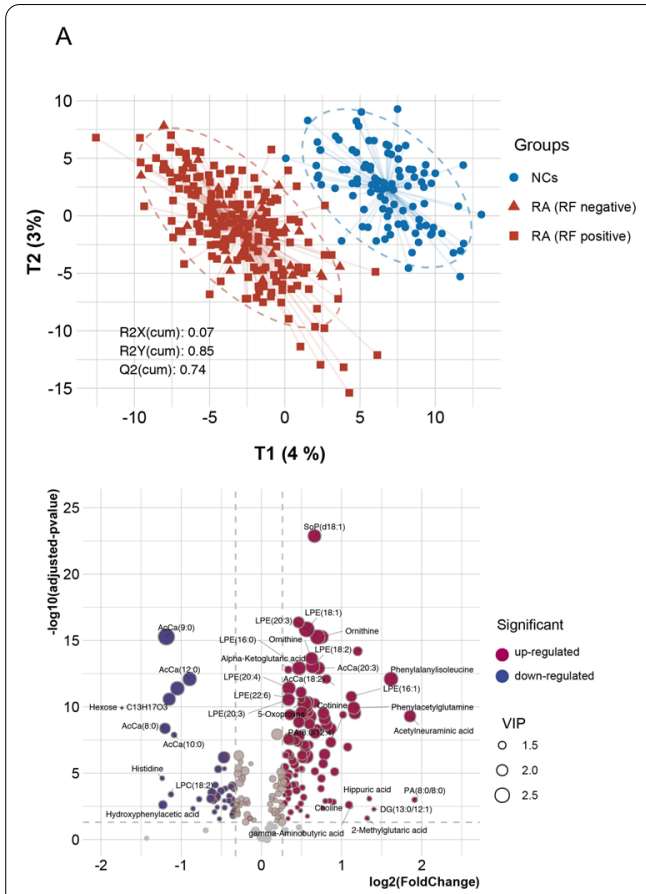

C

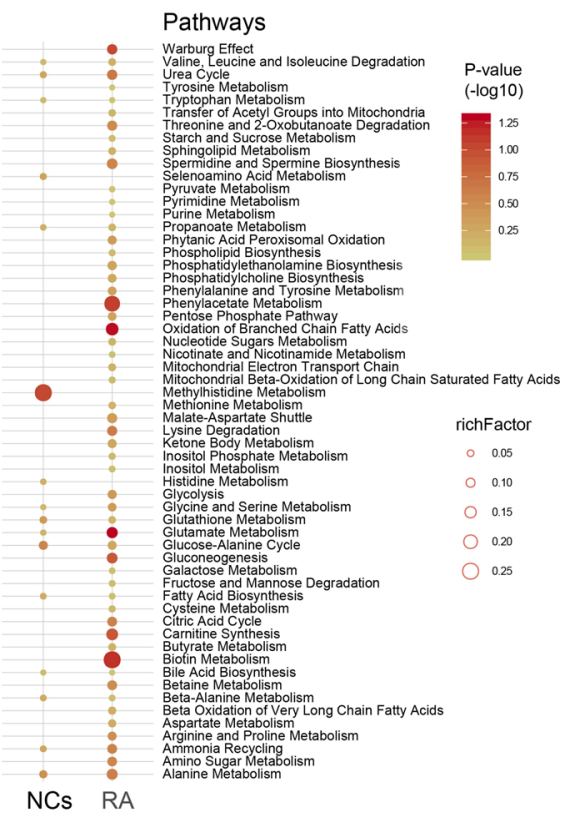

D

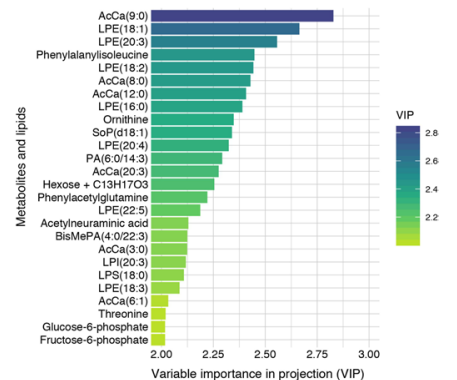

$\mathrm{E}$

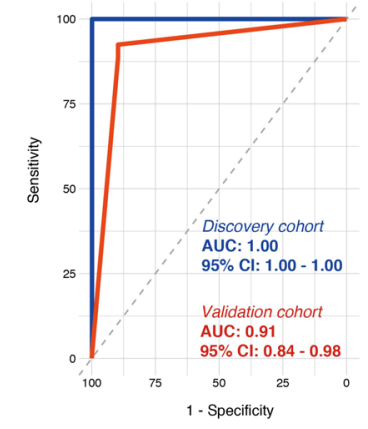

Fig. 2 Metabolomic and lipidomic profiles and multivariate diagnostic model. A A PLS-DA model constructed from metabolomic and lipidomic profiling separated seropositive RA and seronegative RA patients from controls (NCs). B Volcano plot of metabolomic and lipidomic levels of RA versus NCs ( $x$ axis, fold change of RA to NCs; $y$ axis, adjusted $p$ value). Metabolites or lipids with $V I P_{\text {plsda }}>1$, fold change $>1.2$, adjusted $p$-value $<0.05$ are colored in red and those with $V \mid P_{\text {plsda }}>1$, fold change $<0.8$, adjusted $p$-value $<0.05$ in blue. C Metabolic pathway enrichment analysis of RA. D Metabolites and lipids with $V I P_{\text {plsda }}>2$ selected for building a multivariate classification model. E ROC analysis of the multivariate classification model

\section{Association of MLP and clinical parameters}

To understand the association of MLP with clinical parameters of RA, we used a correlation-based network approach to explore the co-occurrence patterns between 28 clinical variables and serum concentrations of 265 identified metabolites and lipids in the RA patients, which could help to explore the correlation of metabolic products and clinical confounders. In the co-occurrence network (Fig. 3), nodes represent clinical parameters or metabolites and lipids, and two nodes are connected if they are significantly correlated (adjusted p-value $<0.05$ and r-value $>0.2$ ). The resulting network consisted of 117 nodes and 274 edges. The modularity index was 0.467 , which is above 0.4 suggested for a modularly structured network [24]. Overall, the entire network could be parsed into six modules. As shown in Fig. 3, module 1 contained CRP, leukocyte, neutrophil and ACPA related to inflammatory activity and 17 metabolites and lipids. Modules 2 and 3 contained RF, IgG, IgM, IgA, globulin, ESR, ANA and AKA related to the immune response and 22 metabolites and lipids. The general clinical variables such as age, gender and BMI were clustered in modules 4-5 and were far away from modules $1-3$. Therefore, the co-occurrence network analysis revealed that there was a clear association between the serum inflammation/ immune markers and concentrations of metabolites and lipids in the RA patients.

\section{Stratification of disease activity using MLP}

To analyze the utility of using MLP for stratification of RA cases based on disease activity, twenty metabolites and lipids strongly correlated to the inflammatory/ immune activity were selected from modules 1 and 2 in the co-occurrence network (Fig. 3). Sixteen metabolites and lipids had significantly increased odd ratios for RA status, while 4 metabolites and lipids had significantly decreased odd ratios (Fig. 4). Disease activity score DAS28-CRP is often utilized to evaluate the disease activity of RA patients [25, 26]. Using DAS28-CRP to stratify RA patients, we further identify 7 metabolites and lipids that were strongly associated with disease activity categories by using the ordinal regression method (Fig. 5). Among these, AcCa (20:3), aspartylphenylalanine (asp-phe), pipecolic acid, lysophosphatidylethanolamine (LPE 18:1) and LPE (20:3) appeared to be positively correlated with higher RA disease activity, while histidine and PA (28:0) were negatively correlated with RA disease activity (Fig. 5A). AcCa (20:3), 


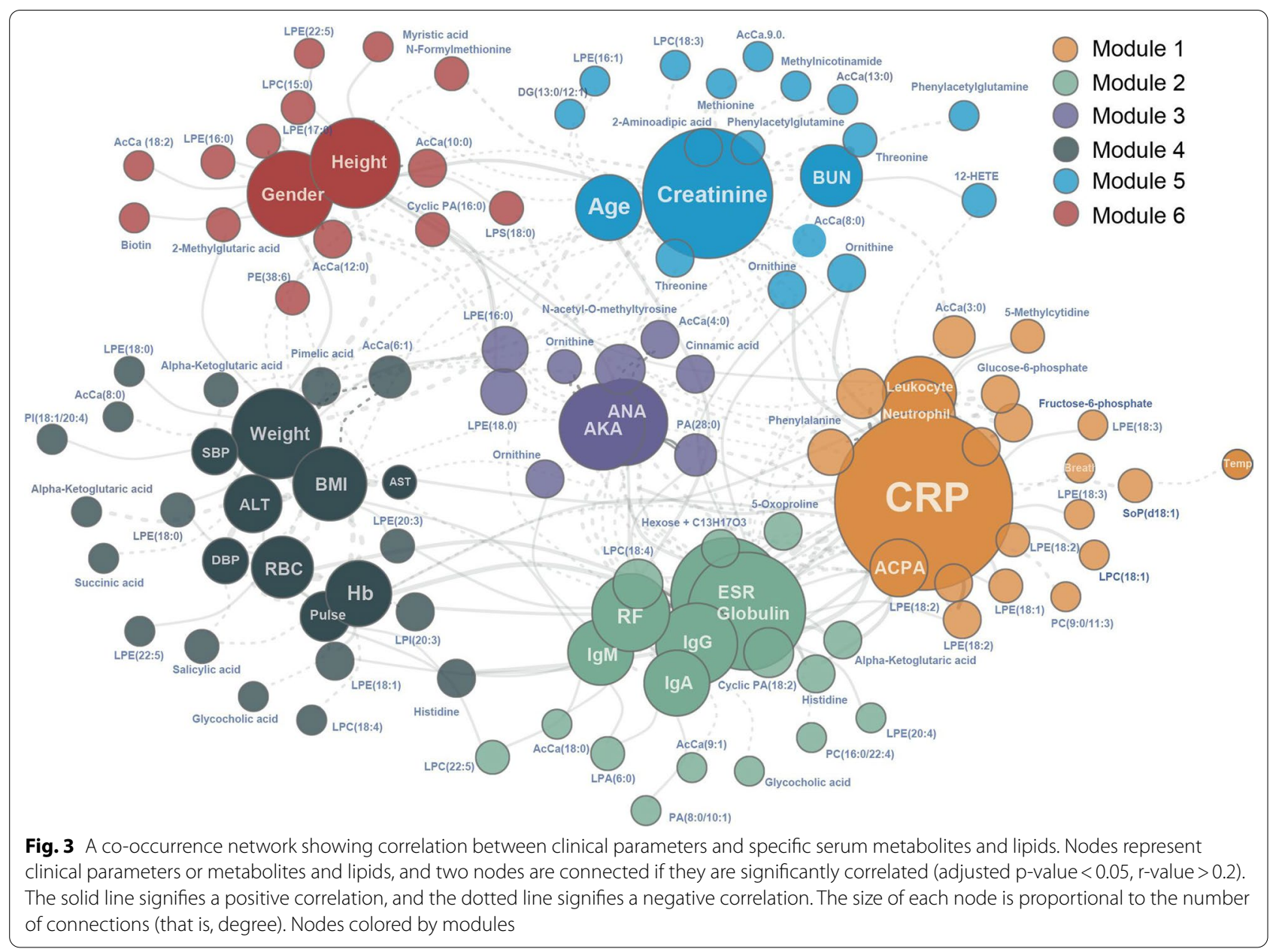

LPE (18:1) and LPE (20:3) were significantly increased in the remission-low risk (R-L) group compared to the NCs group $(\mathrm{p}<0.05$, AUC $>0.7)$. A small peptide aspphe and lipid LPE (18:1) were significantly increased in the DAS28-CRP high disease activity (HIGH) group compared with the R-L group $(\mathrm{p}<0.05$, AUC $>0.7)$, indicating their potential role as biomarkers in RA disease activity stratification (Fig. 5B).

\section{Discussion}

RA is a highly heterogeneous disease with variable presenting symptoms, severity and response to treatment. Autoimmune damage may happen years before symptoms occur and clinical diagnosis is made. It is important for early and accurate diagnosis of RA and prompt initiation of effective treatment to prevent joint damage and functional loss [27]. Current diagnostic criteria are based on comprehensive evaluation of symptoms, serology status and acute phase reactant levels. Because a significant percentage of RA patients are negative for serologic markers, additional diagnostic methods are actively being developed to help increase diagnostic accuracy, particularly for seronegative RA patients. The chronic inflammation and joint destruction in RA patients may cause metabolic perturbations in the peripheral blood, providing opportunities to discover potential biomarkers to improve the clinical diagnosis of RA $[4,28]$.

Previous studies have reported metabolic changes in RA by using nuclear magnetic resonance (NMR) or RPLC-MS [4, 10, 11]. We developed a multi-platform strategy including two reverse phase and two HILIC chromatography hyphenated to high-resolution mass spectrometry (HRMS) in positive and negative ionization modes for the simultaneous quantitative analysis of metabolites and lipids. By means of the integrative metabolomic and lipidomic analysis, we were able to identify 26 serum metabolites and lipids that correctly classified RA patients from normal control subjects. A multivariate classification model was derived from the discovery set and was subsequently validated using an independent validation set. It is noteworthy that our model combined with RF test reached $100 \%$ validation 


A Metablites/Lipids
AMINO ACIDS METABOLISM
5-Oxoproline
Ornithine
Phenylalanine
Histidine
CITRIC ACID CYCLE
Alpha-Ketoglutaric acid
FATTY ACIDS OXIDATION
AcCa(3:0)
GLYCOLYSIS
Fructose-6-phosphate
Glucose-6-phosphate
LIPIDS METABOLISM
12-HETE
Cyclic PA(18:2)
LPC(18:1)
LPC(18:4)
LPE(18:1)
LPE(18:2)
LPE(18:3)
LPI(20:3)
LPS(18:0)
PA(28:0)
PC(9:0/11:3)
S1P

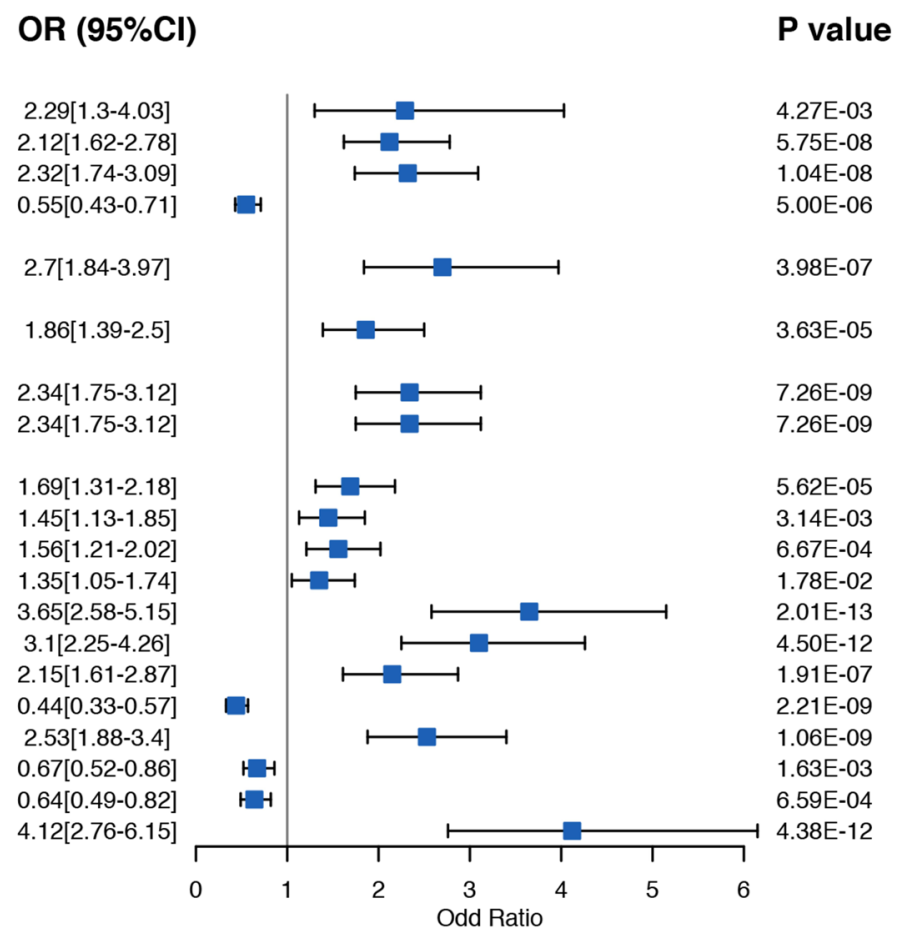

B
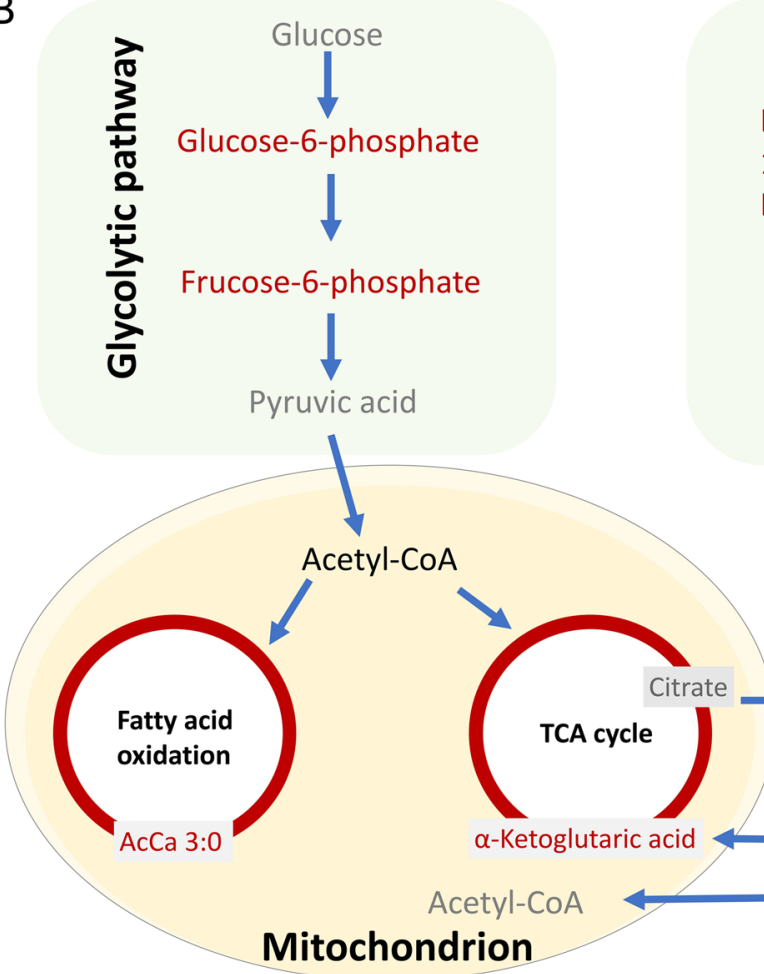

\section{Phospholipids}

LPC 18:1; LPC 18:4; LPE 18:1; LPE 18:2; LPE 18:3; LPE 20:0; LPI 20:3; LPS 18:0; PA 28:0; PC (9:0/11:3)

Sphingomyelin

Sphingosine-1-phosphate

Arachidonic acid

12-hydroxyeicosatetraenoic acid

\section{Lipids metabolism}

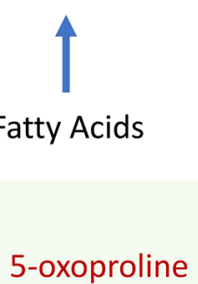

Phenylalanine

\section{Amino acid metabolism}

Fig. 4 Association between the inflammation-immune activity and aberrant metabolism. A Serum metabolites and lipids levels were associated with the risk for RA according to relative peak intensity from untargeted mass spectrometry analyses of subjects $(N=325)$. B RA-associated metabolite and lipids in cellular metabolic pathways. Upregulated metabolites or lipids were colored in red and downregulated metabolites or lipids were colored in green 


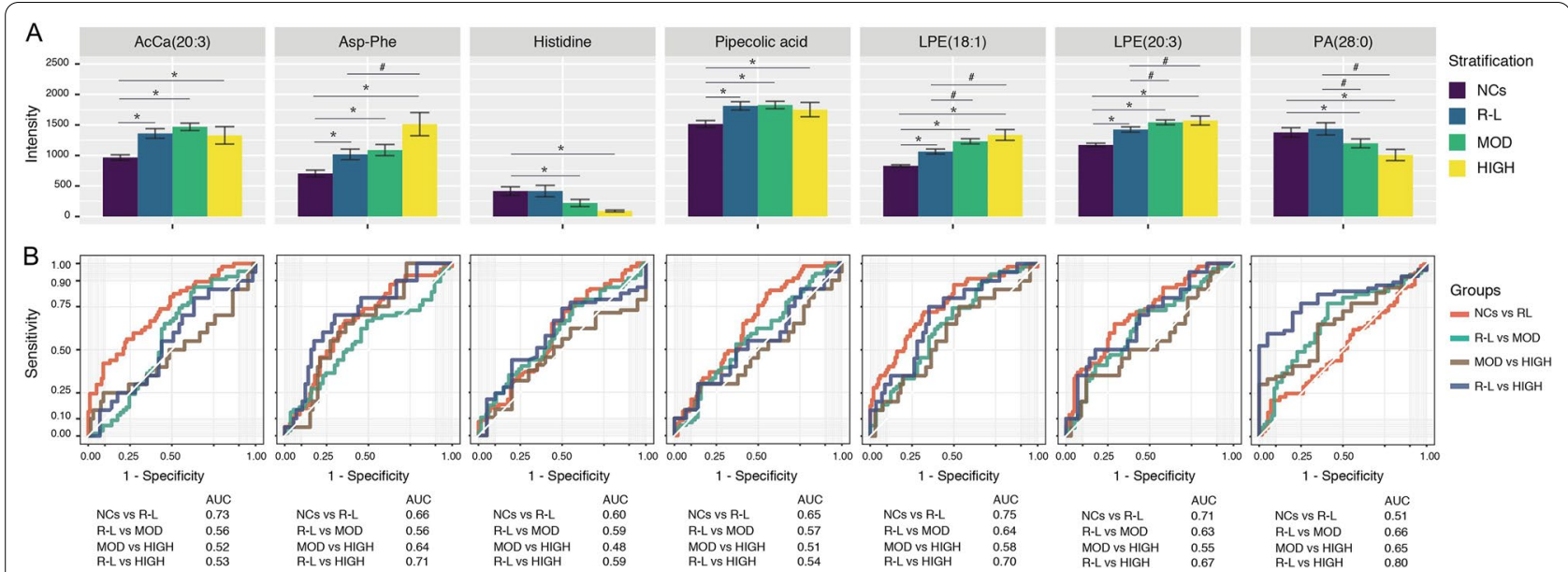

Fig. 5 Metabolites and lipids correlated with RA disease activity. Boxplots (A) and ROC analysis (B) of seven metabolites and lipids with differential levels among normal control group (NCS) and RA with low disease activity (R-L), moderate disease activity (MOD) and high disease activity (HIGH). \# $p<0.05$ versus NCs group, ${ }^{*} p<0.05$ versus $R-L$ group. Data are presented as mean $\pm S E M$, and analyzed by Wilcoxon-Mann $U$ test with FDR control

accuracy, and all patients with seronegative RA were identified by using our model, thus showing the potential for providing a diagnostic tool in both seropositive and seronegative RA.

Similar findings pertaining to the differences in the levels of lysine, phenylalanine, proline, ornithine and salicylic acid between the RA patients and normal control subjects were observed previously $[11,29]$. These metabolites have been associated with immune system activation [4, 30, 31]. On the other hand, we found serum level of histidine, which has anti-inflammatory effects and antioxidative stress effect via inhibiting peroxisome proliferator-activated receptor $\gamma$ (PPAR $\gamma$ ) -involved pathways [32], was significantly decreased in RA patients. The role of histidine in RA needs further studies since histidine supplementation did not show an advantage in a double-blind trial [33]. In our study histidine level was inversely correlated with the RA disease activity defined by DAS28-CRP. Histidine level was significantly decreased in patients with moderate and high disease activity, while no significant changes were seen in RA patients with remission and low disease activity (Fig. 5A).

Lipids are implicated in diverse biological functions, including being the major component of cell membranes and the regulation of cell migration and inflammation [7, 34]. Our study found that increased levels of lipid mediators such as sphingosine 1-phosphate (S1P) and 12-HETE and lysophospholipids such as LPE (18:1), LPE (18:2), lysophosphatidylcholine (LPC 18:1), LPC (18:4) and lysophosphatidylserine (LPS 18:0) in RA patients were positively correlated with CRP levels (Fig. 3), supporting their roles in inflammation. Other groups have reported that LPC was shown to induce cyclooxygenase-2 expression in the vascular endothelial cells, playing a possible pro-inflammatory role [35]. S1P is generated from sphingosine by activation of sphingosine kinase and has been implicated as a potential therapeutic target in RA [36]. Moreira et al. reported that 12-HETE, an arachidonic acid-derived metabolite, may play an important role in the chronic inflammatory process associated with RA by mediating proinflammatory actions [37].

Ongoing inflammation and immune activation are characterized by rising energy demand that is required for immune cell growth, proliferation and the production of proinflammatory molecules [38]. In our study, the increased levels of metabolites (e.g., alpha-ketoglutaric acid, AcCa (3:0), fructose-6-phosphate and glucose6-phosphate) seen in the serum of RA patients indicate enhanced energy metabolism involving glycolysis, citric acid cycle and fatty acid oxidation (Fig. 4). Methotrexate (MTX), a first line treatment for RA, is an antimetabolite drug that targets folic acid metabolism. Many ongoing clinical trials are investigating potential drugs that target lipid/glucose metabolic pathways to improve inflammation control and disease outcome [5]. In addition, using metabolomic approach to delineate inflammation-associated metabolic status of RA patients may offer a promising method for disease activity and treatment effect monitoring.

The strengths of our study include that it employs a comprehensive multi-platform approach for metabolomic and lipidomic analysis, large sample size and its potential to provide diagnostic value for both seropositive and seronegative RA patients. Its limitations include that it is still a discovery study that shows correlation of 
serum markers to RA, but does not directly address the mechanisms of these markers in RA. The diagnostic value of the discovered metabolomic and lipidomic markers for discriminating between seronegative RA and other types of inflammatory arthritis such as psoriatic arthritis, reactive arthritis or spondyloarthritis will be investigated in future studies. For developing our method into clinical practice, our classification model still needs to be evaluated in other multinational/multiethnic cohorts.

In conclusion, we have built a serum metabolic/lipidomic-markers-based model that has potential diagnose value. Our study suggests the integrative comprehensive metabolomic and lipidomic profiling is a promising system biology approach for uncovering biomarkers useful for RA diagnosis and disease activity monitoring.

\section{Supplementary Information}

The online version contains supplementary material available at https://doi. org/10.1186/s12967-021-03169-7.

Additional file 1. Materials and Methods, Quality Control, Tables S1-S3, Figure S1-S3.

\section{Acknowledgements}

The authors would like to acknowledge the financial support from the National Natural Science Foundation of China (Ref. No.: 21904058, Ref. No.: 21904057 and Ref. No.: 81774096), the Open Projects of the Discipline of Chinese Medicine of Nanjing University of Chinese Medicine Supported by the Subject of Academic Priority Discipline of Jiangsu Higher Education Institutions (NO. ZYX03KF031), the Young Elite Scientists Sponsorship Program by CAST (Ref. No.: QNRC2-B04).

\section{Authors' contributions}

HL: Conceptualization, Methodology, Investigation, Writing-Original draft, Writing-Review and editing. WG: Resources. HL: Resources. ZW: Investigation, Writing-Original draft. LL: Resources. MK: Resources. JL: Resources. WC: Resources. ZL: Writing-Review and editing. YX: Writing-Review and editing. JX: Writing-Review and editing. YZ: Writing-Review and editing. ZC: Conceptualization. SL: Resources, Conceptualization. WZ: Analysis, Writing-Review and editing. All authors read and approved the final manuscript.

\section{Funding}

This research received no external funding.

\section{Availability of data and materials}

All data generated or analyzed during this study are included in this published article [and its Additional file].

\section{Declarations}

Ethics approval and consent to participate

The study was approved by the medical ethics committee of the Affiliated Hospital of Nanjing University of Chinese Medicine. Written informed consents were obtained from all study subjects.

\section{Consent for publication}

Written informed consent for publication of their clinical details and/or clinical images was obtained from the patient/parent/guardian/ relative of the patient.

\section{Competing interests}

The authors declare no conflict of interest.

\section{Author details}

${ }^{1}$ School of Medicine, Academy for Advanced Interdisciplinary Studies, Southern University of Science and Technology, 1088 Xueyuan Rd., Shenzhen, China. ${ }^{2}$ Affiliated Hospital of Nanjing University of Chinese Medicine, Nanjing 210029, Jiangsu, China. ${ }^{3}$ Sustech Core Research Facilities, Southern University of Science and Technology, Shenzhen, China. ${ }^{4}$ College of Pharmacy, Jiangsu Collaborative Innovation Center of Chinese Medicinal Resources Industrialization, Nanjing University of Chinese Medicine, Nanjing 210046, China. ${ }^{5}$ State Key Laboratory of Natural Medicines, School of Traditional Chinese Pharmacy, China Pharmaceutical University, Nanjing 210009, China. ${ }^{6}$ State Key Laboratory of Environmental and Biological Analysis (SKLEBA), Department of Chemistry, Hong Kong Baptist University, Kowloon Tong, Hong Kong, China.

Received: 15 August 2021 Accepted: 23 November 2021

Published online: 07 December 2021

\section{References}

1. Bugatti S, Manzo A, Montecucco C, Caporali R. The clinical value of autoantibodies in rheumatoid arthritis. Front Med. 2018;5:1-10. https:// doi.org/10.3389/fmed.2018.00339/full.

2. Saraux A, Berthelot JM, Gérard C, Henaff C, Mary JY, Thorel JB, et al. Value of laboratory tests in early prediction of rheumatoid arthritis. Arthritis Care Res (Hoboken). 2002;47:155-65. https://doi.org/10.1002/art.10241.

3. Wasserman A. Rheumatoid arthritis: common questions about diagnosis and management. Am Fam Physician. 2018;97:455-62.

4. Souto-Carneiro M, Tóth L, Behnisch R, Urbach K, Klika KD, Carvalho RA, et al. Differences in the serum metabolome and lipidome identify potential biomarkers for seronegative rheumatoid arthritis versus psoriatic arthritis. Ann Rheum Dis. 2020;79:499-506. https://doi.org/10.1136/annrh eumdis-2019-216374

5. Chimenti MS, Triggianese $\mathrm{P}$, Conigliaro $\mathrm{P}$, Candi E, Melino G, Perricone R. The interplay between inflammation and metabolism in rheumatoid arthritis. Cell Death Dis. 2015;6:e1887-e1887. https://doi.org/10.1038/ cddis.2015.246

6. Kerekes G, Nurmohamed MT, González-Gay MA, Seres I, Paragh G, Kardos Z, et al. Rheumatoid arthritis and metabolic syndrome. Nat Rev Rheumatol. 2014;10:691-6. https://doi.org/10.1038/nrrheum.2014.121.

7. van Halm VP, Nielen MMJ, Nurmohamed MT, van Schaardenburg D, Reesink HW, Voskuyl AE, et al. Lipids and inflammation: serial measurements of the lipid profile of blood donors who later developed rheumatoid arthritis. Ann Rheum Dis. 2006;66:184-8. https://doi.org/10.1136/ard. 2006.051672.

8. Liao H, Wu J, Kuhn E, Chin W, Chang B, Jones MD, et al. Use of mass spectrometry to identify protein biomarkers of disease severity in the synovial fluid and serum of patients with rheumatoid arthritis. Arthritis Rheum. 2004;50:3792-803. https://doi.org/10.1002/art.20720.

9. Carlson AK, Rawle RA, Wallace CW, Adams E, Greenwood MC, Bothner B, et al. Global metabolomic profiling of human synovial fluid for rheumatoid arthritis biomarkers. Clin Exp Rheumatol. 2019;37:393-9.

10. Dubey D, Kumar S, Chaurasia S, Guleria A, Ahmed S, Singh R, et al. NMRbased serum metabolomics revealed distinctive metabolic patterns in reactive arthritis compared with rheumatoid arthritis. J Proteome Res. 2018. https://doi.org/10.1021/acs.jproteome.8b00439.

11. Li J, Che N, Xu L, Zhang Q, Wang Q, Tan W, et al. LC-MS-based serum metabolomics reveals a distinctive signature in patients with rheumatoid arthritis. Clin Rheumatol. 2018;37:1493-502. https://doi.org/10.1007/ s10067-018-4021-6.

12. Sasaki C, Hiraishi T, Oku T, Okuma K, Suzumura K, Hashimoto M, et al. Metabolomic approach to the exploration of biomarkers associated with disease activity in rheumatoid arthritis. PLoS ONE. 2019;14:e0219400. https://doi.org/10.1371/journal.pone.0219400.

13. Zabek A, Swierkot J, Malak A, Zawadzka I, Deja S, Bogunia-Kubik K, et al. Application of $1 \mathrm{H}$ NMR-based serum metabolomic studies for monitoring female patients with rheumatoid arthritis. J Pharm Biomed Anal. 2016;117:544-50. https://doi.org/10.1016/j.jpba.2015.10.007.

14. Aletaha D, Neogi T, Silman AJ, Funovits J, Felson DT, Bingham CO, et al. 2010 Rheumatoid arthritis classification criteria: An American College of Rheumatology/European League Against Rheumatism collaborative 
initiative. Arthritis Rheum. 2010;62:2569-81. https://doi.org/10.1002/art. 27584.

15. Luan H, Meng N, Liu P, Feng Q, Lin S, Fu J, et al. Pregnancy-induced metabolic phenotype variations in maternal plasma. J Proteome Res. 2014;13:1527-36. https://doi.org/10.1021/pr401068k.

16. Luan H, Liu L-F, Tang Z, Zhang M, Chua K-K, Song J-X, et al. Comprehensive urinary metabolomic profiling and identification of potential noninvasive marker for idiopathic Parkinson's disease. Sci Rep. 2015;5:13888. https://doi.org/10.1038/srep13888.

17. Ji F, Sreenivasmurthy SG, Wei J, Shao X, Luan H, Zhu L, et al. Study of BDE47 induced Parkinson's disease-like metabolic changes in C57BL/6 mice by integrated metabolomic, lipidomic and proteomic analysis. J Hazard Mater. 2019;378:120738. https://doi.org/10.1016/j.jhazmat.2019.06.015.

18. Mahieu NG, Genenbacher JL, Patti GJ. A roadmap for the XCMS family of software solutions in metabolomics. Curr Opin Chem Biol. 2016;30:87-93.

19. Luan H, Jiang X, Ji F, Lan Z, Cai Z, Zhang W. CPVA: A web-based metabolomic tool for chromatographic peak visualization and annotation. Bioinformatics. 2020;2:89.

20. Luan H, Ji F, Chen Y, Cai Z. statTarget: A streamlined tool for signal drift correction and interpretations of quantitative mass spectrometry-based omics data. Anal Chim Acta. 2018;1036:66-72. https://doi.org/10.1016/j. aca.2018.08.002.

21. Chong J, Wishart DS, Xia J. Using MetaboAnalyst $4^{*} 0$ for Comprehensive and Integrative Metabolomics Data Analysis. Curr Protoc Bioinforma. 2019;68:1-128. https://doi.org/10.1002/cpbi.86.

22. Archer KJ, Hou J, Zhou Q, Ferber K, Layne JG, Gentry AE. ordinalgmifs: An $\mathrm{R}$ package for ordinal regression in high-dimensional data settings. Cancer Inform. 2014;13:S20806. https://doi.org/10.4137/CIN.S20806.

23. Robin X, Turck N, Hainard A, Tiberti N, Lisacek F, Sanchez J-C, et al. pROC: an open-source package for $\mathrm{R}$ and $\mathrm{S}+$ to analyze and compare ROC curves. BMC Bioinform. 2011;12:77.

24. Mehrle D, Strosser A, Harkin A. Walk-modularity and community structure in networks. Netw Sci. 2015;3:348-60.

25. Arts EEA, Fransen J, Den Broeder AA, van Riel PLCM, Popa CD. Low disease activity (DAS28 $\leq 3^{*} 2$ ) reduces the risk of first cardiovascular event in rheumatoid arthritis: a time-dependent Cox regression analysis in a large cohort study. Ann Rheum Dis. 2017;76:1693-9. https://doi.org/10.1136/ annrheumdis-2016-210997.

26. Wells G, Becker J-C, Teng J, Dougados M, Schiff M, Smolen J, et al. Validation of the 28-joint Disease Activity Score (DAS28) and European League Against Rheumatism response criteria based on C-reactive protein against disease progression in patients with rheumatoid arthritis, and comparison with the DAS28 based on erythr. Ann Rheum Dis. 2009;68:954-60. https://doi.org/10.1136/ard.2007.084459.

27. Smolen JS, Aletaha D, Barton A, Burmester GR, Emery P, Firestein GS, et al. Rheumatoid arthritis. Nat Rev Dis Prim. 2018;4:18001. https://doi.org/10. 1038/nrdp.2018.1.

28. Tomizawa T, Ito H, Murata K, Hashimoto M, Tanaka M, Murakami K, et al. Distinct biomarkers for different bones in osteoporosis with rheumatoid arthritis. Arthritis Res Ther. 2019;21:174. https://doi.org/10.1186/ s13075-019-1956-1.

29. Kim S, Hwang J, Xuan J, Jung YH, Cha H-S, Kim KH. Global metabolite profiling of synovial fluid for the specific diagnosis of rheumatoid arthritis from other inflammatory arthritis. PLoS ONE. 2014;9:e97501. https://doi. org/10.1371/journal.pone.0097501.

30. Li P, Yin Y-L, Li D, Woo Kim S, Wu G. Amino acids and immune function. Br J Nutr. 2007;98:237-52.

31. Qi Y, Pi Z, Liu S, Song F, Lin N, Liu Z. A metabonomic study of adjuvantinduced arthritis in rats using ultra-performance liquid chromatography coupled with quadrupole time-of-flight mass spectrometry. Mol Biosyst. 2014;10:2617. https://doi.org/10.1039/c4mb00131a.

32. Sun $X$, Feng R, Li Y, Lin S, Zhang W, Li Y, et al. Histidine supplementation alleviates inflammation in the adipose tissue of high-fat diet-induced obese rats via the NF-KB- and PPARY-involved pathways. Br J Nutr. 2014;112:477-85.

33. Pinals RS, Harris ED, Burnett JB, Gerber DA. Treatment of rheumatoid arthritis with L-histidine: a randomized, placebo-controlled, double-blind trial. J Rheumatol. 1977:4:414-9.

34. Navarro-Millán I, Goyal P, Safford MM. Lipid screening and statins alongside disease-modifying anti-rheumatic drugs for patients with rheumatoid arthritis. Rheumatology. 2019;58:933-4.
35. Brouwers H, von Hegedus J, Toes R, Kloppenburg M, loan-Facsinay A. Lipid mediators of inflammation in rheumatoid arthritis and osteoarthritis. Best Pract Res Clin Rheumatol. 2015;29:741-55.

36. Hu P, Chen Y, Cai P, Jiang L, Wu L. Sphingosine-1-phosphate: a potential therapeutic target for rheumatoid arthritis. Mol Biol Rep. 2011;38:422530. https://doi.org/10.1007/s11033-010-0545-9.

37. Moreira V, Gutiérrez JM, Lomonte B, Vinolo MAR, Curi R, Lambeau G, et al. 12-HETE is a regulator of PGE2 production via COX-2 expression induced by a snake venom group IIA phospholipase A2 in isolated peritoneal macrophages. Chem Biol Interact. 2020;317:108903. https://doi.org/10. 1016/j.cbi.2019.108903.

38. Patel $\mathrm{CH}$, Leone RD, Horton MR, Powell JD. Targeting metabolism to regulate immune responses in autoimmunity and cancer. Nat Rev Drug Discov. 2019;18:669-88. https://doi.org/10.1038/s41573-019-0032-5.

\section{Publisher's Note}

Springer Nature remains neutral with regard to jurisdictional claims in published maps and institutional affiliations.
Ready to submit your research? Choose BMC and benefit from:

- fast, convenient online submission

- thorough peer review by experienced researchers in your field

- rapid publication on acceptance

- support for research data, including large and complex data types

- gold Open Access which fosters wider collaboration and increased citations

- maximum visibility for your research: over 100M website views per year

At BMC, research is always in progress.

Learn more biomedcentral.com/submissions 